\title{
Urine Antigen Testing is Equally Sensitive to B. dermatitidis and B. gilchristii Infections
}

Klaire L. Laux, MS; Jennifer L. Anderson, BS; Stephen P. Bentivenga, PhD; Joseph A. McBride, MD; Alana Sterkel, PhD; Eduard Matkovic, MD; Gregory M Gauthier, MD; and Jennifer K. Meece, PhD

Introduction: Blastomycosis is endemic in Wisconsin with Blastomyces dermatitidis and B. gilchristii responsible for infections. Urine antigen testing is a non-invasive diagnostic method for blastomycosis with up to $93 \%$ test sensitivity. However, the test's sensitivity has not been evaluated with relationship to $B$. gilchristii infections.

Methods: We aimed to assess physician use of the urine antigen assay and its sensitivity to B. gilchristii and B. dermatitidis infections in a retrospective study. Culture confirmed clinical cases of blastomycosis from 2008-20I6 were identified within Marshfield Clinic Health System (MCHS) and UW Hospital and Clinics (UWHC) medical records. Clinical data were abstracted from each medical record and included the following: patient demographics, presence of immune compromising and underlying medical conditions, treatment drugs, presence of isolated pulmonary or disseminated disease, death, urine antigen testing, timeframe of testing, and quantitative test values (EIA units or $\mathrm{ng} / \mathrm{mL}$ ).

Results: A total of I40 blastomycosis cases were included in this study, with MCHS contributing II4 cases to the study and UWHC contributing 26 cases. The majority of UWHC cases $(n=22 ; 85 \%)$ were caused by $B$. dermatitidis and the majority of MCHS cases $(n=73 ; 64 \%)$ were caused by $B$. gilchristii. UWHC physicians were significantly more likely to treat with multiple drugs during the course of infection and were more likely to prescribe amphotericin B and voriconazole. Urine antigen testing was more frequently used at UWHC ( $n=24 ; 92 \%)$ than MCHS ( $=51$; 45\%; $P<0.0000 I)$. In this study, the urine antigen assay demonstrated $79 \%$ sensitivity. Sensitivity was significantly associated with the timeframe of testing $(P<$ $0.05)$, with most true positive urine antigen tests ( $83 \%$ ) being performed $\leq 7$ days from diagnosis. In this study, the urine antigen assay was capable of detecting both B. dermatitidis and B. gilchristii at about equal sensitivity. Urine antigen concentration $(\mathrm{ng} / \mathrm{mL})$ trended higher in B. dermatitidis infections.

Conclusion: This study found that the urine antigen assay is capable of detecting both species of Blastomyces at about the same sensitivity. We recommend continued use of the urine antigen assay for diagnosis of blastomycosis and recommend that the assay be used early in the diagnostic process to minimize the chance of false negative results.

Keywords: Blastomycosis; Urine antigen; Blastomyces dermatitidis; Blastomyces gilchristii

Corresponding Author: Jennifer K. Meece, PhD, Marshfield Clinic Research Institute, $1000 \mathrm{~N}$ Oak Avenue, Marshfield, WI 54449, Tel: (7I5) 221-6465, Email: meece.jennifer@marshfieldresearch.org

Financial Support: This work was supported by Marshfield Clinic Research Institute, University of Wisconsin-Oshkosh, Office of Scholarly Research and Creative Activity, and Department of Medicine at the University of Wisconsin - Madison.

Conflict of Interest: G. Gauthier and J. McBride have received funding from MiraVista Diagnostics for an unrelated study.

Ethics: This study was IRB approved with waiver of informed consent.
Received: November 8, 2019

Revised: April 8, 2020

Accepted: April 24, 2020

doi: $10.3121 / \mathrm{cmr} .2020 .1534$ 
$\mathrm{B}$ lastomycosis is a potentially lethal fungal infection caused by inhalation of Blastomyces spp. spores or hyphal fragments. Once inhaled, spores and hyphae undergo a thermally regulated dimorphic switch to yeast. ${ }^{1}$ Clinical manifestations are primarily pulmonary, but extrapulmonary dissemination to other organs is common. ${ }^{2-3}$ Disseminated disease, respiratory failure, and death can occur in both immunocompetent and immunocompromised hosts. Initial clinical presentation of pulmonary blastomycosis resembles viral and/or bacterial pneumonia frequently resulting in a delay of diagnosis, which may be associated with worse clinical outcomes. ${ }^{4}$

At least two Blastomyces spp. cause disease in Wisconsin, USA with $B$. gilchristii more often associated with isolated pulmonary disease and $B$. dermatitidis more likely to disseminate. ${ }^{3,5}$ Despite established associations between Blastomyces spp. and human clinical phenotype, identification of the pathogen to the species level is not routinely performed in clinical diagnostic settings.

The Blastomyces quantitative EIA antigen test (MiraVista Diagnostics, Indianapolis, IN) was developed as an alternative diagnostic method with shorter result turn-around time than fungal culture. ${ }^{6}$ The assay can be performed on multiple specimen types including serum, plasma, urine, cerebrospinal fluid, and bronchoalveolar lavage, although urine is most frequently assayed clinically as it is an easy to obtain sample. In 2015, Frost and Novicki ${ }^{7}$ demonstrated that the quantitative version of the urine antigen test, updated in $2008,{ }^{8-9}$ might be useful to track response to therapy. Currently, we do not have a good understanding of how frequently physicians use the urine antigen assay to diagnose and/or track response to therapy. The sensitivity of the urine EIA antigen test in previous studies has ranged from $76-93 \%,{ }^{6,8,10}$ but the assay was developed prior to the discovery of B. gilchristii as a cryptic species within $B$. dermatitidis. ${ }^{5}$ The test's sensitivity with relationship to $B$. gilchristii infections has not been documented in the literature. We aimed to assess physician use of the urine antigen assay and its sensitivity to $B$. gilchristii and $B$. dermatitidis infections in a retrospective study.

\section{Methods}

Cases

Due to changes to the urine EIA antigen assay in 2008 to make the test quantitative, cases included in this study were from 2008 and after. Culture confirmed clinical cases of blastomycosis from 2008-2016 were identified within Marshfield Clinic Health System (MCHS), Marshfield, WI and University of Wisconsin Hospital and Clinics (UWHC), Madison, WI electronic medical records. Case inclusion criteria were as follows: (1) laboratory confirmed diagnosis of blastomycosis using standard culture methods; (2) Blastomyces clinical isolate for each case was previously bio-banked and available for genotyping; and (3) clinical data were available for abstraction, including patient demographics, urine antigen testing frequency, and quantitative assay results.

\section{Data abstraction}

Clinical data were abstracted as previously described from each medical record. ${ }^{3}$ Demographics included, age at diagnosis, sex, and race/ethnicity. Presence of immune compromising conditions (ICC) included the following: HIV/AIDS diagnosis, transplant recipient within one year, currently receiving chemotherapy, and/or currently receiving immunosuppressive therapy for an autoimmune disease. Underlying medical conditions (UMC) included any ICC(s) listed above and the following conditions: cancer, diabetes, hypertension, hyperlipidemia/hypercholesterolemia, hypothyroidism, COPD, coronary artery disease, asthma, and renal insufficiency/ renal failure. All antifungal drug(s) used during the course of treatment were abstracted for each case patient along with death as an outcome. Patient infections were categorized as isolated pulmonary or disseminated. Disseminated disease was defined as extra-pulmonary infection anywhere outside of, or in addition to, the lungs. We recorded physician use of the urine antigen EIA assay, timeframe of testing ( $\leq 7$ days post diagnosis or $>7$ days post diagnosis), and quantitative test values (EIA units or $\mathrm{ng} / \mathrm{mL}$ ). For MCHS cases, average length of time (days) was calculated for time from symptom onset to urine antigen testing. For the purposes of this manuscript, true positive rate and sensitivity will be used interchangeably.

\section{Isolate genotyping}

Clinical isolates of Blastomyces were archived under IRB approved bio-banking protocols at both Marshfield Clinic Research Institute and UWHC. Blastomyces spp. genotyping was performed for all cases. DNA from each cultured isolate was extracted as previously described. ${ }^{3}$ At Marshfield Clinic Research Institute, species typing of each isolate was performed by either Sanger sequencing or SNP analysis ${ }^{11}$ of the internal transcribed spacer 2 (its2) with species assignment being based on a fixed nucleotide difference between $B$. dermatitidis and B. gilchristii at position 19. ${ }^{5}$ At UWHC, isolates were assigned to species based on a previously established size variation in the promoter region of BAD1.12-13

\section{Statistical analysis}

Study variables including, patient demographics, infecting species, dissemination of disease, treatment, death, physician use and timeframe of the urine antigen EIA assay, and test sensitivity were summarized overall and by Blastomyces spp., study site, and isolated pulmonary or disseminated disease status. Sensitivity of the urine antigen EIA assay was compared by Blastomyces spp., study site, disseminated or isolated pulmonary disease, and testing timeframe. All comparisons were made using Fisher's exact test (Yates correction) with significance defined as $P \leq 0.05$. Urine antigen EIA quantitative values, $\leq 7$ days post diagnosis $(\mathrm{ng} / \mathrm{mL})$, and age at diagnosis were compared using the one-factor ANOVA test for independent measures. Where multiple values were obtained 
Table 1. Study variables by Blastomyces spp., study site, and isolated pulmonary or disseminated disease status.

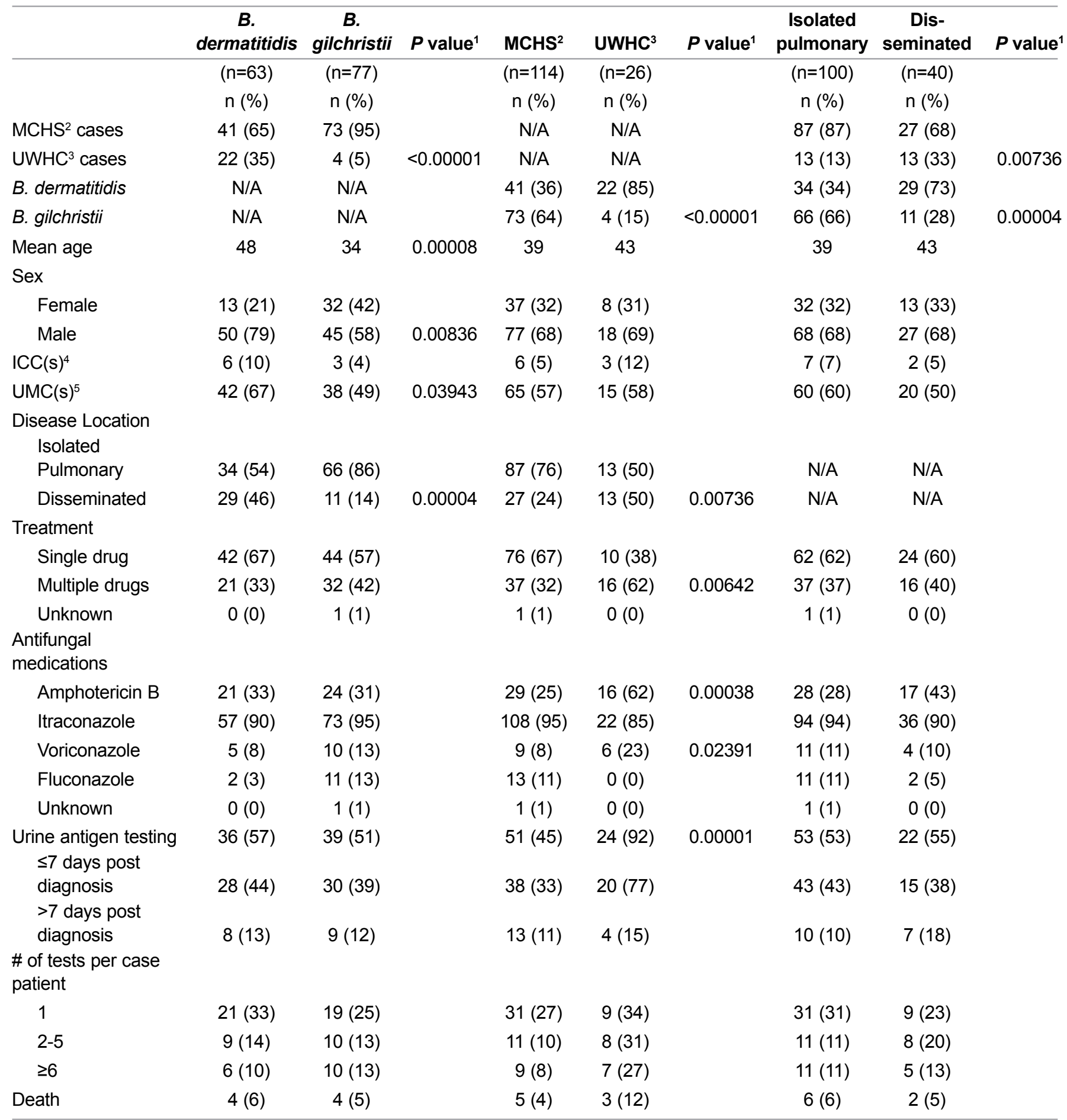

${ }_{1}$ Fisher's exact test (Yates correction) with significance was defined as $P \leq 0.05$. Age at diagnosis was compared using the one-factor ANOVA test for independent measures.

2 Marshfield Clinic Health System

3 University of Wisconsin Hospital and Clinic

${ }^{4}$ Immune compromising conditions included the following: HIV/AIDS diagnosis, transplant recipient within one year, currently receiving chemotherapy, and/or currently receiving immunosuppressive therapy for an autoimmune disease

5 Underlying medical conditions included any ICC(s) listed above and the following conditions: cancer, diabetes, hypertension, hyperlipidemia/ hypercholesterolemia, hypothyroidism, COPD, coronary artery disease, asthma, and renal insufficiency/renal failure 
$\leq 7$ days post-diagnosis on the same patients, only the initial positive value was used for this comparison. Values of $>14.7$ (above limit of detection) were assessed as 14.7 for ANOVA modeling.

\section{Results}

A total of 140 cases of blastomycosis, diagnosed from 20082016, were included in this study. MCHS contributed 114 cases to the study, with UWHC contributing 26 cases. Enrollment cases represented approximately $30 \%$ of total cases seen annually at both sites individually. $B$. dermatitidis was the cause of 63 cases (45\%), and B. gilchristii was the cause of 77 cases $(55 \%)$. The age of patients at diagnosis ranged from 3-93 years (median 39) and 95 (68\%) were male. $\mathrm{UMC}(\mathrm{s})$ and ICC(s), as defined in the methods section above, were identified in $79(56 \%)$ and $9(9 \%)$ patients, respectively, with data being unknown due to lack of medical history in 11 patients. Patients identified themselves by the following racial groups: non-Hispanic White $(n=103 ; 74 \%)$, Hispanic White $(n=6 ; 4 \%)$, African American $(n=2 ; 1 \%)$, Asian $(n=13$; $9 \%)$, American Indian $(n=7 ; 5 \%)$, unknown $(n=7 ; 5 \%)$, White, unknown ethnicity $(n=1 ; 1 \%)$, and biracial, nonHispanic White and African American ( $\mathrm{n}=1 ; 1 \%)$. Disseminated disease occurred in 40 cases (29\%). Eight cases $(6 \%)$ resulted in death. Itraconazole was the most frequently prescribed drug, with $93 \%$ of patients $(n=130)$ receiving it at some point in their illness. Fifty-three patients (38\%) received multiple antifungal medications throughout the course of treatment. Urine antigen EIA testing was ordered by physicians in 75 cases $(54 \%)$, with multiple tests (2-18 tests/ case patient; average 6) performed in 35 cases $(25 \%)$. Of those patients tested, most were tested within $\leq 7$ days postdiagnosis $(n=58 ; 77 \%)$ and received only one test $(n=40$; $53 \%$ ). Within the 75 true cases for which urine antigen EIA testing was performed, 16 cases were negative, demonstrating a $79 \%$ overall sensitivity rate.

Table 2. Urine antigen testing by study site over study timeframe

\begin{tabular}{ccccc}
\hline & \multicolumn{2}{c}{ MCHS $^{1}$} & \multicolumn{2}{c}{ UWHS $^{2}$} \\
& $\mathbf{n}$ & $(\%)$ & $\mathbf{n}$ & $(\%)$ \\
\hline 2008 & 1 & $(7)$ & $\mathrm{N}^{\prime} \mathrm{A}^{3}$ & $\mathrm{~N} / \mathrm{A}^{3}$ \\
2009 & 5 & $(26)$ & $\mathrm{N} / \mathrm{A}^{3}$ & $\mathrm{~N} / \mathrm{A}^{3}$ \\
2010 & 12 & $(38)$ & 2 & $(100)$ \\
2011 & 4 & $(57)$ & 1 & $(50)$ \\
2012 & 9 & $(64)$ & 2 & $(100)$ \\
2013 & 12 & $(75)$ & 1 & $(100)$ \\
2014 & 5 & $(71)$ & 4 & $(100)$ \\
2015 & 2 & $(67)$ & 5 & $(100)$ \\
2016 & 1 & $(25)$ & 7 & $(88)$ \\
2017 & $\mathrm{~N} / \mathrm{A}^{3}$ & $\mathrm{~N} / \mathrm{A}^{3}$ & 2 & $(100)$
\end{tabular}

${ }^{1}$ Marshfield Clinic Health System

2 University of Wisconsin Hospital and Clinic

${ }^{3}$ No samples included from study site for that year
$B$. dermatitidis cases were more often associated with older age, male sex, and presence of UMC than those caused by $B$. gilchristii (Table 1). In addition, $B$. dermatitidis infections were more likely to cause disseminated disease than $B$. gilchristii infections. Fifty-eight cases (28 B. dermatitidis cases and $30 \mathrm{~B}$. gilchristii cases) had initial urine antigen EIA testing within $\leq 7$ days post diagnosis. Of those, only $21 B$. dermatitidis cases and $14 \mathrm{~B}$. gilchristii cases had quantitative values in $\mathrm{ng} / \mathrm{mL}$ within $\leq 7$ days post diagnosis for comparison, with the remaining being reported in EIA units. EIA quantitative values for $B$. dermatitidis infections ranged from 0.41 to $>14.7$ (average 5.72) and for B. gilchristii ranged from 0.29 to 10.3 (average 2.68) (Supplemental data available online). For these tests, ANOVA analysis revealed no significant difference in initial positive urine antigen concentration by infecting Blastomyces spp., although antigen concentration trended higher in $B$. dermatitidis infections than $B$. gilchristii infections $(P=0.0519$; Figure 1$)$. Testing timeframe (days) was also calculated with reference to symptom onset for MCHS cases only. Within the 38 case patients from MCHS that were tested within $\leq 7$ days post diagnosis, the average time from symptom onset to testing was 28 days (range 5-89, standard deviation [SD] 20, median 22). Within the 13 MCHS patients tested $>7$ days post diagnosis, time from symptom onset to testing varied greatly ranging from 23 to 519 days (average 137, SD 137, median 100) for the following reasons: delayed diagnosis, rule out of reoccurrence, or use of the assay for testing at the midpoint or endpoint of treatment.

Cases caused by the two species were significantly different $(P<0.00001)$ by study site with the majority of UWHC cases $(85 \%, \mathrm{n}=22)$ being caused by $B$. dermatitidis and the majority of MCHS cases $(64 \%, \mathrm{n}=73)$ being caused by B. gilchristii (Table 1). UWHC also had significantly more cases of disseminated disease as compared to MCHS. UWHC physicians were significantly more likely to treat with multiple drugs during the course of infection and were more likely to prescribe amphotericin B and voriconazole, with MCHS physicians observationally using fluconazole more than UWHC. Urine antigen testing was more frequently used at UWHC $(\mathrm{n}=24,92 \%)$ than MCHS $(\mathrm{n}=51,45 \% ; P<$ 0.00001 , Table 1$)$, which did not vary much over the years of the study (Table 2). UWHC physicians also tended to test earlier and more frequently throughout treatment; presumably to track response to therapy.

In this study, the urine antigen assay was capable of detecting both $B$. dermatitidis and $B$. gilchristii at about the same sensitivity (Table 3 ), with no difference in test sensitivity by species or study site. Sensitivity trended higher in isolated pulmonary cases versus disseminated cases. Sensitivity was significantly associated with the time of testing $(P=0.0071)$, with most true positive urine antigen EIA tests (83\%) being performed $\leq 7$ days of diagnosis. In addition, false negative test results trended higher in cases of disseminated disease 


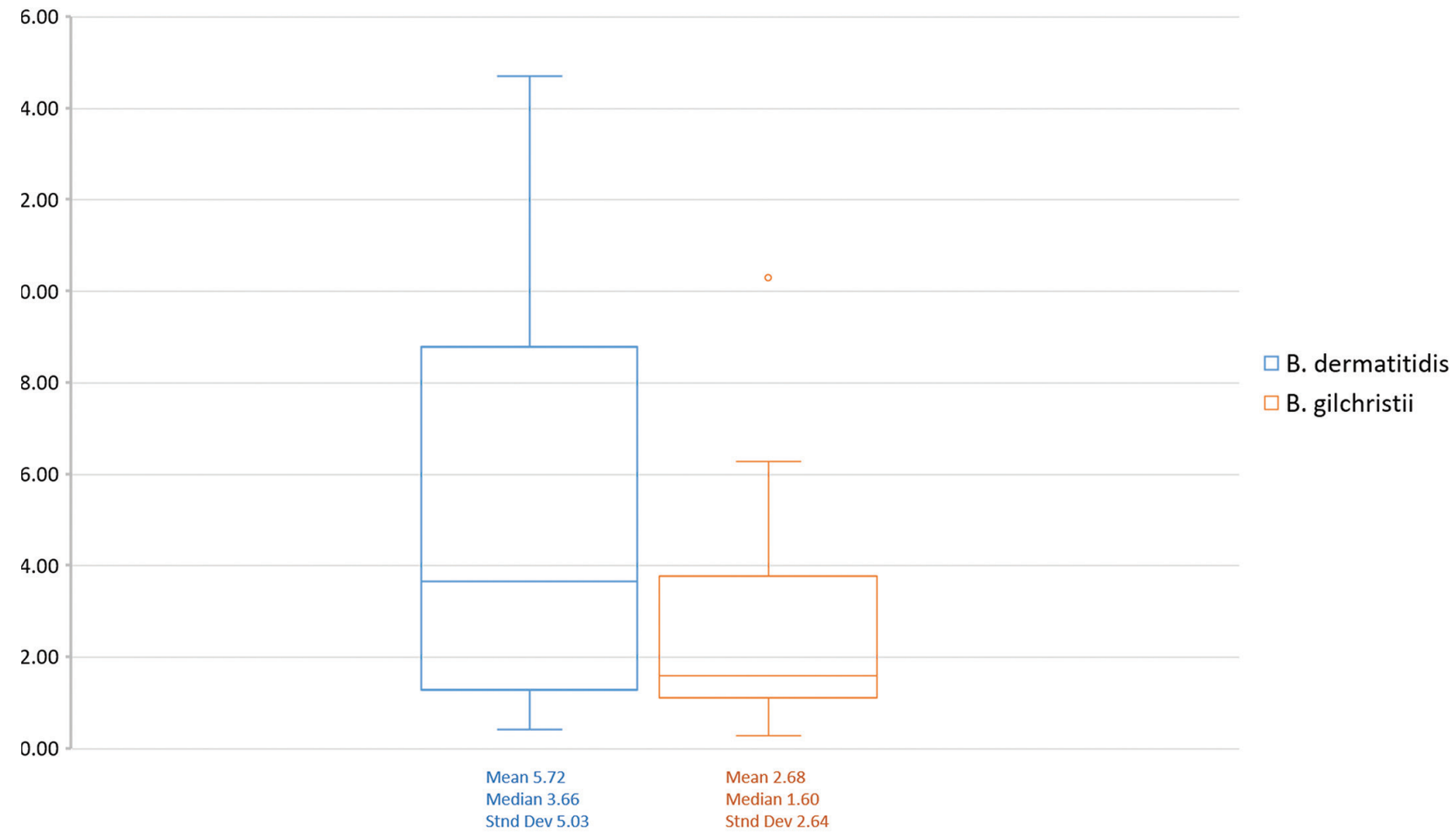

Figure 1. Urine antigen EIA concentration ( $\mathrm{ng} / \mathrm{mL}, \leq 7$ days of diagnosis) by $B$. gilchristii and $B$. dermatitidis infections compared using the one-factor ANOVA test for independent measures. The interquartile range box represents the middle $50 \%$ of date with the line inside the box representing the median. Upper and lower whiskers extend from the minimum to maximum data point with outlier data indicated as a dot beyond the whisker.

(Table 3), but this did not seem to be due to disseminated cases being tested later in the course of disease (Table 1).

\section{Discussion}

Despite the usefulness of the urine antigen EIA test, until now the sensitivity of the assay to detect $B$. gilchristii infections had not been evaluated. We aimed to assess physician use of the urine antigen assay and its sensitivity to $B$. gilchristii and $B$. dermatitidis infections independently in a retrospective study.

An unexpected finding in this study was that the Blastomyces spp. varied significantly by study site, with cases at UWHC more frequently caused by $B$. dermatitidis. This may be due to slightly different, although overlapping, endemic regions of $B$. gilchristii and $B$. dermatitidis as previously described by Brown et al. ${ }^{5}$ Although in order to truly understand the geographic distributions of these two pathogens, it would be necessary to obtain geo-coded exposure locations for individual case patients. UWHC also saw significantly more disseminated disease than MCHS, likely due to previously established association between $B$. dermatitidis and disseminated disease, ${ }^{3}$ that are corroborated by this study (Table 1).
In this study, physicians used the urine antigen assay in about half $(54 \%)$ of blastomycosis cases, although UWHC physicians used it at a much higher rate than MCHS physicians. UWHC physicians also tended to use the test earlier in the disease course ( $\leq 7$ days post-diagnosis) and more frequently throughout treatment, presumably to monitor disease progress and response to therapy. In addition, UWHC physicians were more likely to prescribe multiple antifungal drugs over the course of treatment and used amphotericin B and voriconazole more frequently than did MCHS physicians. Fluconazole use trended higher in MCHS cases. It is important to note that the small number of cases included from UWHC could, inflate any study site differences we observed. Alternatively, case patients that present at UWHC may be more likely to be seen by a specialty physician (due to the size of the health system and potential for complex case referrals) who may more routinely use urine antigen testing and change antifungal drug based on response to therapy than a primary care physician.

We observed comparable urine antigen EIA test sensitivity to previous published studies, although much variability has been observed across studies. ${ }^{5-10}$ As we observed in the current study, testing timeframe had a significant impact on 
the assay's sensitivity and may be one of the reasons for reported variations in sensitivity. As reflected by a closer look at time from symptom onset to testing in patients with testing $>7$ day from diagnosis in only MCHS cases, this category reflects cases with significant delays in diagnosis. Several of these previous studies had samples obtained "at the time of initial diagnosis/newly diagnosed." ${ }^{\prime, 14}$ However, the present study includes cases in which testing was delayed. A more recent study comprised mostly of Wisconsin case patients reported $87.9 \%$ sensitivity, but when sensitivity was calculated based on culture positive cases (positive predictive value), as was done in the present study, sensitivity was $76.3 \%{ }^{15}$

We found that the urine antigen assay is capable of detecting both species of Blastomyces at about the same sensitivity. This may have been somewhat expected due to its previously recognized cross-reactivity of the assay to Histoplasma capsulatum. B. gilchristii and $B$. dermatitidis ostensibly are more antigenically similar to each other than to $H$. capsulatum and therefore sensitivity should be equivalent between species. Nonetheless, this was a clinically relevant question that directly impacts the diagnosis of this important fungal disease so pursuing it was crucial.

The concentration of urine antigen in patients infected with $B$. dermatitidis trended higher than patients infected with $B$. gilchristii. Although this finding was not significant, we feel that our analysis may have been limited by the small number of urine antigen positive case patients, with testing performed $\leq 7$ days of diagnosis in this study $(n=35)$. Higher antigen concentration in patients infected with $B$. dermatitidis could indicate differences in pathogenicity. Previous studies have shown that $B$. dermatitidis infections are more likely to cause disseminated disease. Perhaps the more systemic nature of these infections results in higher antigen detection in the urine. A larger study would be necessary to determine if urine antigen concentration is significantly different in B. gilchristii and $B$. dermatitidis infection and what infection and host factors influence it. In addition, it is not clear how physicians interpret antigen concentration values or its clinical relevance on disease progression. We plan to explore these questions further in future studies.

We believe this study has several strengths. Access to blastomycosis cases from two separate health care systems provided enough statistical power to detect a difference across most variables assessed in this study. The demographic and clinical data available on these cases allowed for description and evaluation of diagnostic practices not previously described. That being said, this study was limited by the retrospective nature. Furthermore, our statistical power was limited to assess significance in some comparisons due to the small number of cases. Despite this, we provide the first evaluation of the sensitivity of the urine antigen EIA test to $B$. dermatitidis and B. gilchristii infections.

Table 3. Sensitivity of the Urine Antigen Assay

\begin{tabular}{|c|c|c|c|}
\hline Species & $\begin{array}{c}\mathrm{n}(\%) \\
\text { B. dermatitidis }\end{array}$ & $\begin{array}{c}\text { n (\%) } \\
\text { B. gilchristii }\end{array}$ & $P$ value ${ }^{1}$ \\
\hline True positives & $26(72)$ & $31(79)$ & \\
\hline False negatives & $10(28)$ & $7(18)$ & \\
\hline Unknown & $0(0)$ & $1(3)$ & \\
\hline Total cases & $36(100)$ & $39(100)$ & \\
\hline Study site & MCHS & UWHC & \\
\hline True positives & $38(75)$ & $19(79)$ & \\
\hline False negatives & $12(24)$ & $5(21)$ & \\
\hline Unknown & $1(2)$ & $0(0)$ & \\
\hline Total cases & $51(100)$ & $24(100)$ & \\
\hline Disease location & Isolated pulmonary & Disseminated & \\
\hline True positives & $43(81)$ & $14(64)$ & \\
\hline False negatives & $9(17)$ & $8(36)$ & \\
\hline Unknown & $1(2)$ & $0(0)$ & \\
\hline Total cases & $53(100)$ & $22(100)$ & \\
\hline Testing timeframe & $\leq 7$ days post-diagnosis & $>7$ days post-diagnosis & \\
\hline True positives & $48(83)$ & $9(53)$ & \\
\hline False negatives & $9(16)$ & $8(47)$ & 0.0071 \\
\hline Unknown & $1(2)$ & $0(0)$ & \\
\hline Total cases & $58(100)$ & $17(100)$ & \\
\hline
\end{tabular}

${ }^{1}$ Fisher's exact test (Yates correction) with significance defined as $P \leq 0.05$. 


\section{Conclusion}

This study found that the urine antigen assay is capable of detecting both species of Blastomyces at about the same sensitivity. We recommend continued use of the urine antigen assay for diagnosis of blastomycosis and recommend that the assay be used early in the diagnostic process to minimize the chance of false negative results.

\section{Acknowledgements}

This work was supported by the Marshfield Clinic Research Institute. Additional funding was provided through the University of Wisconsin Oshkosh, Office of Scholarly Research and Creative Activity and Department of Medicine at the University of Wisconsin-Madison. A special thanks to Dr. Mathew Hall for consulting on this study, and especially for help understanding how physicians may use urine antigen testing. Additional thanks to Patrick Stockwell and Steve Kaiser for laboratory support.

\section{References}

1. Drutz DJ, Frey CL. Intracellular and extracellular defenses of human phagocytes against Blastomyces dermatitidis conidia and yeasts. J Lab Clin Med. 1985;105(6):737-750.

2. Pappas PG, Dismukes WE. Blastomycosis: Gilchrist's disease revisited. Curr Clin Top Infect Dis. 2002;22:61-77.

3. Meece JK, Anderson JL, Gruszka S, Sloss BL, Sullivan B, Reed KD. Variation in clinical phenotype of human infection among genetic groups of Blastomyces dermatitidis. J Infect Dis. 2013;207(5):814-822.

4. Chapman SW, Dismukes WE, Proia LA, et al; Infectious Diseases Society of America. Clinical practice guidelines for the management of blastomycosis: 2008 update by the Infectious Diseases Society of America. Clin Infect Dis. 2008;46(12):1801-1812.

5. Brown EM, McTaggart LR, Zhang SX, Low DE, Stevens DA, Richardson SE. Phylogenetic analysis reveals a cryptic species Blastomyces gilchristii, sp. nov. within the human pathogenic fungus Blastomyces dermatitidis. PLoS One. 2013;8(3):e59237.

6. Durkin M, Witt J, LeMonte A, Wheat B, Connolly P. Antigen assay with the potential to aid in diagnosis of blastomycosis. $\mathrm{J}$ Clin Microbiol. 2004;42(10):4873-4875.

7. Frost HM, Novicki TJ. Blastomyces Antigen Detection for Diagnosis and Management of Blastomycosis. J Clin Microbiol. 2015;53(11):3660-3662.

8. Connolly P, Hage CA, Bariola JR, et al. Blastomyces dermatitidis antigen detection by quantitative enzyme immunoassay. Clin Vaccine Immunol. 2012;19(1):53-56.

9. Hage CA, Davis TE, Egan L, et al. Diagnosis of pulmonary histoplasmosis and blastomycosis by detection of antigen in bronchoalveolar lavage fluid using an improved secondgeneration enzyme-linked immunoassay. Respir Med. 2007;101(1):43-47.

10. Carlos WG, Rose AS, Wheat LJ, et al. Blastomycosis in Indiana: digging up more cases. Chest. 2010;138(6):13771382.

11. Frost HM, Anderson JL, Ivacic L, Sloss BL, Embil J, Meece JK. Development and validation of a novel single nucleotide polymorphism (SNP) panel for genetic analysis of Blastomyces spp. and association analysis. BMC Infect Dis. 2016;16(1):509.
12. Burgess JW, Schwan WR, Volk TJ. PCR-based detection of DNA from the human pathogen Blastomyces dermatitidis from natural soil samples. Med Mycol. 2006;44(8):741-748.

13. Meece JK, Anderson JL, Klein BS, et al. Genetic diversity in Blastomyces dermatitidis : implications for PCR detection in clinical and environmental samples. Med Mycol. 2010;48(2):285-290.

14. Bariola JR, Hage CA, Durkin M, et al. Detection of Blastomyces dermatitidis antigen in patients with newly diagnosed blastomycosis. Diagn Microbiol Infect Dis. 2011;69(2):187-191.

15. Baumgardner DJ. Use of urine antigen testing for Blastomyces in an integrated health system. J Patient Cent Res Rev. 2018;5(2):176-182.

\section{Author Affiliations}

Klaire L. Laux, MS*; Jennifer L. Anderson, BS*; Stephen P. Bentivenga, PhD ; Joseph A. McBride, MD $\$$; Alana Sterkel, PhD\|, $\mid$; Eduard Matkovic, MD\|; Gregory M Gauthier, MDt; Jennifer K. Meece, $P h D^{*}$

*Integrated Research and Development Laboratory, Marshfield Clinic Research Institute, Marshfield Clinic Health System, Marshfield, WI 54449

†Department of Biology, University of Wisconsin Oshkosh, Oshkosh, WI 54901

\$Division of Infectious Disease, Department of Medicine, University of Wisconsin School of Medicine and Public Health, Madison, WI 53792

$\S$ Division of Infectious Disease, Department of Pediatrics, University of Wisconsin School of Medicine and Public Health, Madison, WI 53792

$\|$ Department of Pathology and Laboratory Medicine, University of Wisconsin School of Medicine and Public Health, Madison, WI 53792

$\uparrow$ Communicable Disease Division, Wisconsin State Laboratory of Hygiene, Madison, WI 53792 